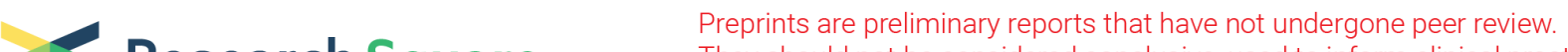 Research Square They should not be considered conclusive, used to inform clinical practice, or referenced by the media as validated information. \\ Chemoprevention of Pancreatic Cancer by Inhibition of Glutathione-S Transferase P1
}

\section{Takahiro Kogawa}

Cancer institute hospital of Japanese foundation for cancer research

Yasushi Sato ( $\nabla$ sato.yasushi@tokushima-u.ac.jp )

Tokushima University Graduate School of Medicine https://orcid.org/0000-0002-6776-4609

\section{Rai Shimoyama}

Shonan Kamakura General Hospital: Shonan Kamakura Sogo Byoin

\section{Wataru Kurata}

Shonan Kamakura General Hospital: Shonan Kamakura Sogo Byoin

\section{Yasuyuki Tashiro}

Shonan Kamakura Sogo Byoin

\section{Yoshiro Niitsu}

Shonan Kamakura General Hospital: Shonan Kamakura Sogo Byoin

\section{Research Article}

Keywords: GSTP1 inhibitor, chemoprevention, pancreatic cancer, rat model, KRAS signal

Posted Date: May 18th, 2021

DOl: https://doi.org/10.21203/rs.3.rs-408015/v1

License: (c) (i) This work is licensed under a Creative Commons Attribution 4.0 International License. Read Full License 


\section{Abstract}

Pancreatic cancer is among the most refractory malignancies with poor prognosis. Thus, preventive approaches, in addition to the development of novel therapeutic strategies are essential for this type of cancer. KRAS mutations occur very early in the development of pancreatic cancers and could be targeted for its prevention, yet specific inhibitors for mutated KRAS are lacking. Accordingly, Glutathione-S Transferase p1 (GSTP1), which we recently found to be an autocrine stimulator of mutated KRAS signaling, is predicted to be an alternative target for chemoprevention of pancreatic cancer. In this study, chemopreventive effects of O-Hexadecyl-y-glutamyl-S-benzyl-cysteinyl-D-phenyl glycine-Ethylester (HGBPE), which we previously synthesized to inhibit GSTP1 activity, was analyzed for its effect on the prevention of a rat pancreatic carcinogenesis model induced by 7,12-dimethyl-benzanthracene (DMBA). Rats administered with DMBA were grouped into five cohorts. In the treated group I, which was treated neither with HGBPE nor vehicle, sequential appearance of precancerous lesions, ductal complexes, and adenocarcinoma was confirmed as previously reported. We also confirmed in this group that mutations of KRAS and expression of GSTP1 simultaneously occurred in the ductal complex. To rats of groups II and IV, HGBPE was administered, and vehicle to those of group III and V. In groups of II and IV, the incidence of both ductal complex and adenocarcinoma were significantly lower than those in groups III and V. These data clearly suggest the efficacy of HGBP as a potential chemopreventive agent for pancreatic cancer.

\section{Introduction}

Pancreatic cancer is often asymptomatic and usually diagnosed at very advanced stages, engendering low survival rates [1]. Thus, except for surgical resection at early stages, therapeutic approaches are largely limited, and the poor prognosis and refractory nature of this disease indicate an increased need for preventive approaches [2]. Preventive measures for pancreatic cancer may include passive prophylaxis; avoidance of risk factors such as tobacco smoke, obesity, type II diabetes, chronic inflammation of the pancreas, high dietary intake of fried food and red meat [3] or active prophylaxis; surgical resection of precancerous legions, IPMN [4], proactive intake of foods containing chemopreventive compounds such as curcumin, vitamins such as vitamins $D$ and $E$, capsaicin, genistein, isothiocyanates [3] and administration of synthetic chemopreventive agents such as NSAIDs, angiotensin-I-converting enzyme inhibitors, aspirin, Ibuprofen, Metformin, Statins, Farnesyl transferase inhibitors [3].

Amongst, the use of chemopreventive agents is a most promising and preferable approach because of its noninvasive nature and availability of efficacy monitoring of cancer at early stages or precancerous lesions during the treatment period. However, to date, there are no conclusive outcomes regarding the clinical efficacy of chemopreventive agents on pancreatic cancers, probably because their previously examined target molecules are not directly linked to the pathogenesis of pancreatic cancer so that their effects are difficult to interpret. The pathogenesis of pancreatic cancer at initial stages often primarily involves KRAS mutations [5] to which direct inhibitory agents are currently unavailable. We have recently 
disclosed that in KRAS mutated cancer cells, glutathione-s transferase P1 (GSTP1) is induced via RAF/MEK/ERK pathway as a down-stream product, which in turn interacts with CRAF to enhance the signaling by forming an autocrine loop [6]. Thus, in mutated KRAS (mKRAS) cells, silencing of GSTP1 expression brought about significant suppression of their in vitro and in vivo growth [6]. Furthermore, in GSTp1 null mice, which exhibited no apparent physical abnormalities, mKRAS relevant colon carcinogenesis evoked by administering azoxymethane was significantly impaired [6], suggesting the potential efficacy of interfering with GSTP1 can be effective chemopreventive agents for mKRAS cancers.

On that basis, chemopreventive effects of O-Hexadecyl-y-glutamyl-S-benzyl-cysteinyl-D-phenylglycineEthylester (HGBPE), which we previously synthesized and proven to be a specific inhibitor of GSTP1 [7], on pancreatic cancer in a rat model was determined in this study.

\section{Materials And Methods}

\section{Rats Animals}

Five-week-old SPF/VAF Crl:CD(SD) rats were purchased from Charles River Japan Inc., (Tokyo, Japan). They were bred under SPF conditions in SPF building, the Animal Research Center, Sapporo Medical University under circumstances of standard food and water. All experiments were undertaken following criteria outlined in a license granted under the Animals (Scientific Procedures) Act 1986 and approved by the Animal Ethics Committees of the Sapporo Medical University.

\section{Development of pancreatic cancer in rats by DMBA}

According to the methods reported earlier [8], S.D. rats underwent laparotomy to create a $5 \mathrm{~mm}$-sized pocket on the pancreatic head and in which a pancreas carcinogen (powdered 7,12-

diethylbenz[a]anthracene) (DMBA) (Sigma Chemical, St. Louis, MO) was embedded in followed by a suture with 6-0 Prolene thread (Fig 1A, B). The amount of DMBA was determined to be $5 \mathrm{mg}$ so that an adenocarcinoma will develop in 9 months with a probability of $30 \%$, according to a previous report [8]. Rats were euthanized by rapid inhalation of diethyl ether, and their pancreas were excised to identify ductal formation and adenocarcinoma.

\section{Chemoprevention study}

Sixty rats underwent embedding of DMBA in their pancreas head to induce the carcinogenesis process, and the animals were grouped into five categories (Fig1.C). Group I consisted of 20 rats was used to monitor the carcinogenesis steps by sacrificing each three rats every 4 week and five rats at final week 24. For group II (10 rats), $20 \mathrm{mg} / \mathrm{kg}$ of HGBPE was administered daily into the abdominal cavity for four weeks, starting 12 weeks after the embedding of DMBA. For group III (10 rats), the vehicle was administered daily into the abdominal cavity for four weeks, starting 12 weeks after DMBA embedding. For group IV (20 rats), $20 \mathrm{mg} / \mathrm{kg}$ of HGBPE was administered daily into the abdominal cavity for six weeks, starting 12 weeks after DMBA embedding. For group V (20 rats), the vehicle was 
administered daily into the abdominal cavity for six weeks, starting 12 weeks after DMBAembedding (Fig 1C). The applied dose was determined to be $20 \mathrm{mg} / \mathrm{kg}$ to attain blood levels to become 50-100 uM so that GSTP1 could be inhibited in vivo according to pharmacokinetic analysis [7].

\section{Identification of ductal complex and adenocarcinoma}

Isolated pancreas tissue samples were attached to a corkboard, positioning its head and tail in a straight line and soaked in $10 \%$ formalin for $24 \mathrm{~h}$ for fixation. The pancreas tissue samples were dissected horizontally into 1-mm length specimens, which were stained for H.E. after paraffin embedding. Ductal complexes, noninvasive precancerous lesions, and adenocarcinomas were identified as described previously [8]. The diagnosis was performed confirmed consistently by two expert animal pathologists, E.T and S.T.

\section{Immunostaining of GSTP1}

Antigen retrieval was performed by autoclave treatment at $100^{\circ} \mathrm{C}$ for 10 min using Target retrieval solution High P.H. (DAKO Cytomation, Kyoto, Japan). At first, GST-pi polyclonal antibody (MBL, Nagoya, Japan), COX-2 polyclonal antibody (Cayman Chemical, Chicago, IL), P53 polyclonal antibody (FL393) (Santa Cruz, Santacruz, CA), and Smad4 monoclonal antibody (B-8) (Santa Cruz) was used. Proteins were identified with Envision kit (DAKO cytomation, Kyoto, Japan). They were colored by 3,3Diaminobenzidine Tetrahydrochloride (DAB) (WAKO, Osaka, Japan), and their nuclei were stained by Gill's hematoxylin solution (WAKO). It was determined to be positive when more than $50 \%$ of the composing cells was stained.

\section{Analysis of KRAS point mutations at codon 12}

Tissue specimens of pancreas ductal complexes and adenocarcinomas were harvested using a laser capture microdissection system, LM100 (Olympus Corporation, Tokyo, Japan), in a microdissection manner, followed by DNA extraction with DNeasy Tissue Kit (QIAGEN, Valencia, CA). According to the method described by Levi et al. et al. [9], the detection of point mutations on K-ras in these specimens was performed by 2-step PCR-restriction enzyme fragment length polymorphism (RFLP). In brief, DNA samples underwent the first PCR reaction using 5'actgagtataaacttgtggtagttggacctg3' (sense) 5 'cagcatttacctctatcgtaggatc3' (antisense) as primers then digested with Mval, restriction enzyme. The subsequent second PCR reaction using 5'acttgtggtagttggaggtg3' (sense) and 5'attcagaatcactttgtgga3' (antisense), digestion was performed on the samples, followed by treatment with $\mathrm{Hphl}$, restriction enzyme. Finally, RFLP analysis was conducted on polyacrylamide gels, 15/25 (Daiichi pure chemical, Tokyo, Japan).

\section{GST-pi specific inhibitor, HGBPE}

GSTP1 specific inhibitor, (01-Hexadecyl-y-glutamyl-S-benzylcysteinyl-D-phenylglycine-Ethylester (HGBPE) (Fig. 1B), which shows in vivo bioavailability was synthesized according to the method described earlier [7]. As HGBP is lipophilic, it was dissolved in a mixture of benzyl-alcohol and ethanol (1:4) and filtered 
through a $1.20 \mu \mathrm{M}$ filter and added to fat preparations to emulsify the preparation (Intralipos) (Otsuka Pharmaceutical, Tokyo, Japan) (1:0.075) to become emulsion before in vivo use.

\section{Statistical processing}

Categorical variables were compared using the Fisher exact test. A P-value of 0.05 was considered significant. All statistical analyses were performed using GraphPad Prism 8 (GraphPad Software, La Jolla, CA).

\section{Results}

\section{Histological confirmation of ductal complex and adenocarcinoma in the pancreas of rat sacrificed at 24th weeks of DMBA implantation}

To validate pancreatic carcinogenesis in the DMBA rat model, pancreas tissue of rats were sacrificed at the 24th week of DMBA implantation were histologically examined. Macroscopic inspection on pancreas specimens, which were sliced into 1-mm width after formalin-fixation and HE staining, revealed whitish nodules in Sect. $0,1,2,3,4,5$ of pancreas head near the DMBA implanted sites, but not in Sect. 6-33 of body and tail of the pancreas (Fig. 2A). Microscopically, in all specimens from Sect. $0-5$, both ductal complex and adenocarcinoma were colocalized. Representing histological images of ductal complex and adenocarcinoma areas in these nodules are shown in Fig. 2B and D, respectively, with each corresponding higher magnification appearance in the rectangle of Fig. $2 \mathrm{C}, \mathrm{E}$.

In the ductal complex area, its histological characteristics, as reported earlier [8]; multiple conglomerates of noninvasive microtubular structures, consisted of cells showing scanty mitosis, enlarged and condensed nucleus nuclei, and cytoplasmic atypia with associated inflammation and fibrosis were verified. Adenocarcinoma lesions were featured by massive invasive cells with aggregated nucleus nuclei of condensed chromatin, pseudostratification, and frequent mitosis (Fig. 2D, E) as reported previously [8].

\section{Incidence of ductal complex and adenocarcinoma lesions during carcinogenesis induced by DMBA}

Taking the histopathological characteristics of ductal complex and adenocarcinoma described above into account, both lesions during carcinogenesis were surveyed in the pancreas excised every 4 weeks after DMBA-implanting in group I rats (Fig. 1C), and the incidence of lesions was counted by defining the lesion as positive when at least one lesion was identified throughout the fields of one specimen. The first appearance of ductal complex occurred at week 16 of DMBA-implantation, with an incidence of $1 / 3$ (33\%); the incidence increased as the stage advanced; $2 / 3(67 \%)$ at week 20 and $4 / 5(80 \%)$ at week 24 . In contrast, adenocarcinoma was seen only at a late stage, week 24 , with an incidence of $3 / 5(60 \%)$ (Table 1). 
Table 1

Incidence of ductal complex and adenocarcinoma (group I)

\begin{tabular}{|lll|}
\hline Week & \begin{tabular}{l} 
Ductal complex \\
\cline { 2 - 3 }
\end{tabular} & Adenocarcinoma \\
\hline 4 & $0 / 3(0)$ & $0 / 3(0)$ \\
\hline 8 & $0 / 3(0)$ & $0 / 3(0)$ \\
\hline 12 & $0 / 3(0)$ & $0 / 3(0)$ \\
\hline 16 & $1 / 3(33)$ & $0 / 3(0)$ \\
\hline 20 & $2 / 3(67)$ & $0 / 3(0)$ \\
\hline 24 & $4 / 5(80)$ & $3 / 5(60)$ \\
\hline
\end{tabular}

Close relationship between KRAS point mutation and GSTP1 expression in ductal complex and adenocarcinoma induced by DMBA

Since the close correlation of KRAS mutation with high expression of GSTP1 in various human cancer tissues and cancer cells, including those of pancreas origin has been well described [6][9], we intended to confirm that this correlation is also found in our pancreas cancer model. In group 1 rats (Fig. 1C), a total of 7 ductal complex specimens; 1, 2, and 4 specimens from 16th week, 20th week, 24th week rats, respectively (Table 1) and 3 adenocarcinoma specimens from 24th week rats (Table 1) were microdissected and underwent for analyses of KRAS mutation at codon 12 by 2-step PCR-restriction enzyme fragment length polymorphism (RFLP). As control samples, 5 specimens from the pancreas head of 16 week-old normal rats were also analyzed for their KRAS mutations by RFLP.

In addition to bands with 75bp mobility, which were found in all control samples, those with $46 \mathrm{bp}$ mobility representing mutated KRAS were clearly identified in samples of ductal complex and adenocarcinoma (Fig. $3 \mathrm{~A}$ ). Incidences of $46 \mathrm{bp}$ band in samples of control, ductal complex, and adenocarcinoma were $0 / 5,5 / 7$, and $3 / 3$, respectively (Fig. $3 B$ ).

When these results were analyzed along the carcinogenesis stages, mutation of KRAS become positive after the 20th week of DMBA implantation; for ductal complex, $1 / 2$ at week 20 and $4 / 4$ at week 24 and for adenocarcinoma, $3 / 3$ at 24 weeks (Table 2). GSTP1 expression in these lesions of group I rats were then examined by immunostaining. Representative staining patterns are shown in Fig. 4. In clear contrast to staining of HE specimens; Fig. 4A, D, G and negative controls which stained with non-specific antibody, those of both duct epithelium of ductal complex (Fig. 4E) and cancerous cell of adenocarcinoma (Fig. 4H) from rats implanted with DMBA for 24 weeks, showed distinct positive staining for GSTP1. 
Table 2

Incidence of mutated KRAS, GSTP1 expression in ductal complex and adenocarcinoma (Group1)

\begin{tabular}{|c|c|c|c|c|}
\hline & \multicolumn{2}{|c|}{ No (week) } & $\begin{array}{l}\text { Mutated } \\
\text { KRAS }\end{array}$ & GSTP1 expression \\
\hline \multirow{6}{*}{$\begin{array}{l}\text { Normal pancreas } \\
\text { tissue }\end{array}$} & 1 & & - & - \\
\hline & 2 & & - & - \\
\hline & 3 & & - & - \\
\hline & 4 & & - & - \\
\hline & 5 & & - & - \\
\hline & & & $0 / 5(0 \%)$ & $0 / 5(0 \%)$ \\
\hline \multirow[t]{8}{*}{ Ductal complex } & 1 & (16) & - & - \\
\hline & 2 & $(20)$ & - & + \\
\hline & 3 & $(20)$ & + & + \\
\hline & 4 & $(24)$ & + & + \\
\hline & 5 & $(24)$ & + & + \\
\hline & 6 & (24) & + & + \\
\hline & 7 & $(24)$ & + & + \\
\hline & & & $5 / 7(71.4 \%)$ & 6/7 (85.7\%) \\
\hline \multirow[t]{4}{*}{ Adenocarcinoma } & 1 & $(24)$ & + & + \\
\hline & 2 & $(24)$ & + & + \\
\hline & 3 & $(24)$ & + & + \\
\hline & & & $3 / 3(100 \%)$ & $3 / 3(100 \%)$ \\
\hline
\end{tabular}

Then, immunostaining analyses of GSTP1 were conducted along carcinogenesis stages, defining it as positive when staining patterns shown in Fig. $4 \mathrm{E}$ or $\mathrm{H}$ were evident in any area of specimens. While all specimens from normal pancreas were negative for staining, those of ductal complex from rats after 20 weeks of DMBA treatment were positive; $2 / 2$ at 20th week and $4 / 4$ at 24th week and those of adenocarcinoma at 24th week were all positive; $3 / 3$ (Table 2).

Effect of HGBPE on the generation of ductal complex and adenocarcinoma at 16 weeks (II, III group) and 36 weeks (IV, V group) after DMBA-embedding 
Incidence of ductal complex and adenocarcinoma was examined after administrating HGBPE to DMBA embedded rats. At week 16 of DMBA-embedding, incidences of the ductal complex in group III (control) that was administrated only vehicle and in group II that was administrated HGBPE were $50 \%(5 / 10)$ and $10 \%(1 / 10)$ respectively, suggesting significant suppression $(p=0.05)$ of ductal complex formation by HGBPE (Table 3). Adenocarcinoma was not developed in both groups. At 36 weeks of DMBA-embedding, incidences of the ductal complex in V group and in IV group were $72.2 \%(13 / 18)$ and in $25.0 \%(5 / 20)$, respectively, with a statistical significance of $p=0.0036$ (Table 3). Further, incidences of adenocarcinoma in $\mathrm{V}$ group and in IV group were 50.0\% (9/18) and15.0\% (3/20) respectively with significant difference ( $\mathrm{p}$ $=0.0247$ ). Collectively, these results suggest that GSTP1 inhibitor, HGBPE is a potent chemopreventive agent against DMBA induced pancreas cancer.

Table 3

Preventive effect of HGBPE on generation of ductal complex and adenocarcinoma (Group II-V)

\begin{tabular}{|lllllll|}
\hline \multicolumn{3}{|c}{ Ductal complex } & \multicolumn{5}{c|}{ Adenocarcinoma } \\
\hline Week & HGBPE & Vehicle & p & HGBPE & Vehicle & P \\
16 & $1 / 10$ & $5 / 10$ & 0.05 & $0 / 10$ & $0 / 10$ & \\
& $(10.0 \%)$ & $(50.0 \%)$ & & $(0.0 \%)$ & $(0.0 \%)$ & \\
\hline 36 & $5 / 20$ & $13 / 18$ & 0.0036 & $3 / 20$ & $9 / 18(50.0 \%)$ & 0.02 \\
& $(25.0 \%)$ & $(72.2 \%)$ & & $(15.0 \%)$ & & \\
\hline \multicolumn{4}{|l}{ HGBPE, 01-Hexadecyl-y-glutamyl-S-benzylcysteinyl-D-phenylglycine-Ethylester } \\
\hline
\end{tabular}

\section{Discussion}

For chemoprevention study, it may be preferable to recruit those subjects at high risk of the disease. As for pancreas cancer, members of pancreatic-cancer-prone families [1], including those with a history of Peutz-Jeghers syndrome, hereditary pancreatitis, and familial atypical multiple mole melanoma (FAMMM) could be, in this regards, ideal candidates for the study. However, the limited population of these subjects and discordance of their genetic background to that of ordinally pancreatic cancer suggest that these members are practically inappropriate populations for the study.

In this regard, those subjects with detectable precancerous legions may be another preferable group as monitoring the legions which could be eradicated by chemopreventive agents in a relatively short term, makes the study more efficient than waiting for a long-term survival outcome in apparently healthy subjects. This short-term treatment with the drug is not only efficient as a study plan but also beneficial for the target subjects because the shorter the treatment period, the less toxicity of the drug is generally guaranteed in clinical trials. 
We have, in fact, previously demonstrated that employing anti-inflammatory drugs, Sulindac and Etodolac, aberrant crypt foci (ACF), which are supposedly precursors of colon polyp-cancer sequence of humans [10], can be useful surrogate legions for chemoprevention of human colon cancer since after two months active treatment, numbers of ACF and adenomas were significantly suppressed at two and twelve months, respectively [10]. We also conducted a similar short term study using sulindac in patients with a certain type predictor of pancreatic malignancy; branch duct-intraductal papillary mucinous neoplasms (BD-IPMNs), and demonstrated that both branch duct diameter and mural nodule height of BD-IPMNs in the treatment group were significantly reduced, while those in the control group were unchanged [11]. Based on these studies, we, in the current study, treated DMBA rats with HGBPE for 4-6 weeks only during the period of the precancerous legion, ductal complex, prior to the appearance of adenocarcinoma and successfully proved the preventive effects on carcinogenesis.

Another critical issue of chemoprevention of cancer is the "efficacy with safety" of the drugs used since accrual subjects are not cancer patients but healthy subjects, and thus, any severe adverse effects caused by drugs are not acceptable. This could be achieved by inhibiting the specific molecule which is directly responsible for carcinogenesis, namely oncogene products. However, regarding pancreas cancer, there is no specific inhibitor at present to the most prevalent oncogene, mKRAS. In this regard, our most recent findings that GSTP1, which is specifically induced by mKRAS in turn, sustains its growth signaling by interacting with CRAF, and that in GSTP1 null mice, mutant KRAS (mKRAS) relevant colon carcinogenesis was significantly impaired [6]. Strongly suggest that inhibitor of GSTP1 could be an ideal agent for chemoprevention of pancreas cancer.

In accordance with this notion, present results suggest the efficacy of GSTP1 inhibitor in the pancreas cancer carcinogenesis model where mutation rate of KRAS and positive rate of GSTP1 expression in the precancerous ductal complex was reasonably high; $71.4 \%$ and $85.7 \%$, respectively. Conversely, the fact that the rate of KRAS mutation and that of GSTP1 positivity were not $100 \%$, possibly because not all carcinogenesis processes in this model are caused by mKRAS, may rationalize the incomplete efficacy of the treatment by the GSTP1 inhibitor (see Table 3). Incidentally, the effects of NSAIDs, sulindac in the above studies, may also be ascribed to their inhibitory activity to GSTP1 because ACF, adenoma, and cancers in colon cancer patients [12], and hyperplasia and adenoma and carcinoma portions of resected pancreatic tissues from BD-IPMN patients were highly positive for GSTP1 but not for cyclooxygenase-1 and -2 which have been believed to be target molecules for Sulindac [11]. Moreover, direct binding of sulindac to GSTP1 has been proven [12].

Nonetheless, although the present study suggests that the use of GSTP1 inhibitor to prevent pancreas cancer is a promising option for its practical application to humans, more detailed explorations on its biochemical and biological properties such as bioavailability, toxicity, cost/benefit issue, etc. are certainly required.

\section{Declarations}




\section{Funding}

Not applicable

\section{Availability of data and material}

All date analyzed in this study are included in this article.

\section{Code availability}

Not applicable

\section{Authors' contributions}

Data curation: KT, YS, RS, WK, and YT, Formal analysis: KT, Supervision: $Y N$, and $Y S$, Investigation: $K T$, Writing-original draft: $Y N$, and $Y S$, Writing-review and editing: $Y N$, and $Y S$

\section{Ethics approval andconsent to participate}

All experiments were undertaken in accordance with criteria outlined in a license granted under the Animals (Scientific Procedures) Act 1986 and approved by the Animal Ethics Committees of the Sapporo Medical University.

\section{Consent for publication}

The participant has consented to the submission of this report to the journal.

\section{Competing interests}

The authors declare no potential conflicts of interest.

YT is an employee of GlaxoSmithKline K.K. This work was conducted independently of, and was not funded by GlaxoSmithKline K.K.

\section{Acknowledgments}

We thank Drs. T. Abe and K. Miyanishi (department of medical oncology, Sapporo medical university school of medicine) for various valuable suggestions for experimental details and technical advice for GSTP1 staining, respectively.

\section{References}

1. Kamisawa T, Wood LD, Itoi T, Takaori K (2016) Pancreatic cancer. The Lancet 388:73-85

2. Miller MS, Allen P, Brentnall TA et al (2016) Pancreatic Cancer Chemoprevention Translational Workshop: Meeting Report. In: Pancreas.1080-1091 
3. Stan SD, Singh SV, Brand RE (2010) Chemoprevention strategies for pancreatic cancer. Nat Rev Gastroenterol Hepatol 7:347-356

4. Benzel J, Fendrich V (2018) Chemoprevention and Treatment of Pancreatic Cancer: Update and Review of the Literature. Digestion 97:275-287

5. Guo S, Shi X, Shen J et al (2020) Preoperative detection of KRAS G12D mutation in ctDNA is a powerful predictor for early recurrence of resectable PDAC patients. $\mathrm{Br} \mathrm{J}$ Cancer 122:857-867. https://doi.org/10.1038/s41416-019-0704-2

6. Niitsu Y, Sato Y, Takanashi K et al (2020) A CRAF/glutathione-S-transferase P1 complex sustains autocrine growth of cancers with KRAS and BRAF mutations. Proc Natl Acad Sci U S A 117:1943519445

7. Nakajima T, Takayama T, Miyanishi K et al (2003) Reversal of multiple drug resistance in cholangiocarcinoma by the glutathione S-transferase- $\pi$-specific inhibitor 0 1-hexadecyl-y-glutamyl-Sbenzylcysteinyl-D-phenylglycine ethylester. J Pharmacol Exp Ther 306:861-869. https://doi.org/10.1124/jpet.103.052696

8. Jimenez RE, Z'graggen K, Hartwig W et al (1999) Immunohistochemical characterization of pancreatic tumors induced by dimethylbenzanthracene in rats. Am J Pathol 154:1223-1229. https://doi.org/10.1016/S0002-9440(10)65374-6

9. Levi S, Urbano-Ispizua A, Gill R et al (1991) Multiple K-ras codon 12 mutations in cholangiocarcinomas demonstrated with a sensitive polymerase chain reaction technique. Cancer Res 51:3497-3502

10. Takayama T, Nagashima H, Maeda M et al (2011) Randomized double-blind trial of sulindac and etodolac to eradicate aberrant crypt foci and to prevent sporadic colorectal polyps. Clin Cancer Res 17. https://doi.org/10.1158/1078-0432.CCR-10-2395

11. Hayashi $T$, Ishiwatari $H$, Ihara $H$ et al (2009) Suppressive effect of sulindac on branch ductintraductal papillary mucinous neoplasms. J Gastroenterol 44:964-975. https://doi.org/10.1007/s00535-009-0089-8

12. Nobuoka A, Takayama T, Miyanishi K et al (2004) Glutathione-S-transferase P1-1 protects aberrant crypt foci from apoptosis induced by deoxycholic acid. Gastroenterology 127:428-443. https://doi.org/10.1053/j.gastro.2004.05.021

\section{Figures}


A
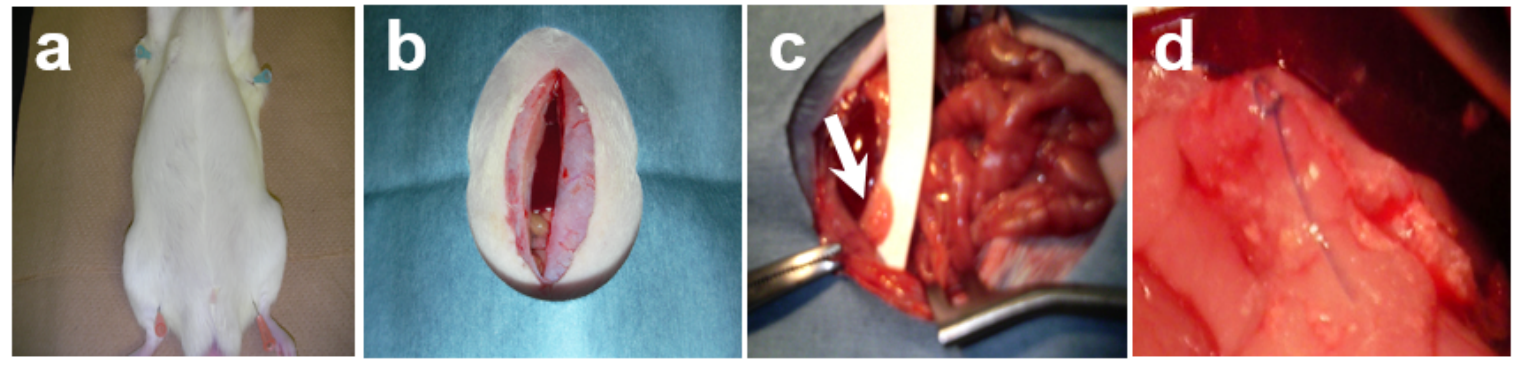

B

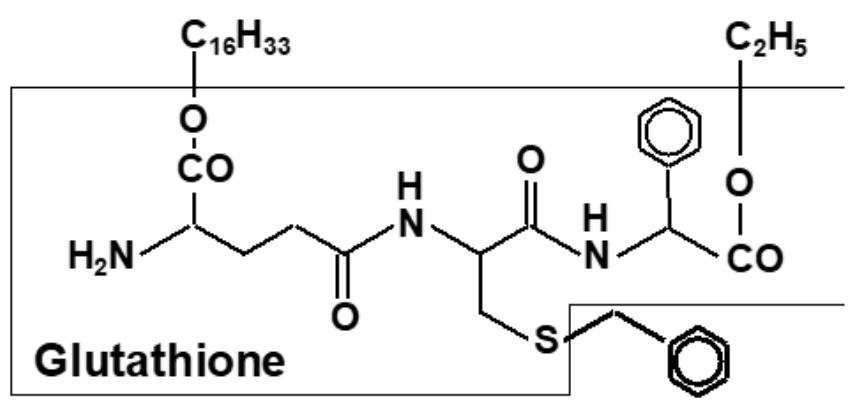

HGBPE, O1-Hexadecyl- $\gamma$-glutamyl-S-benzylcysteinyl-D-phenylglycine-Ethylester

C

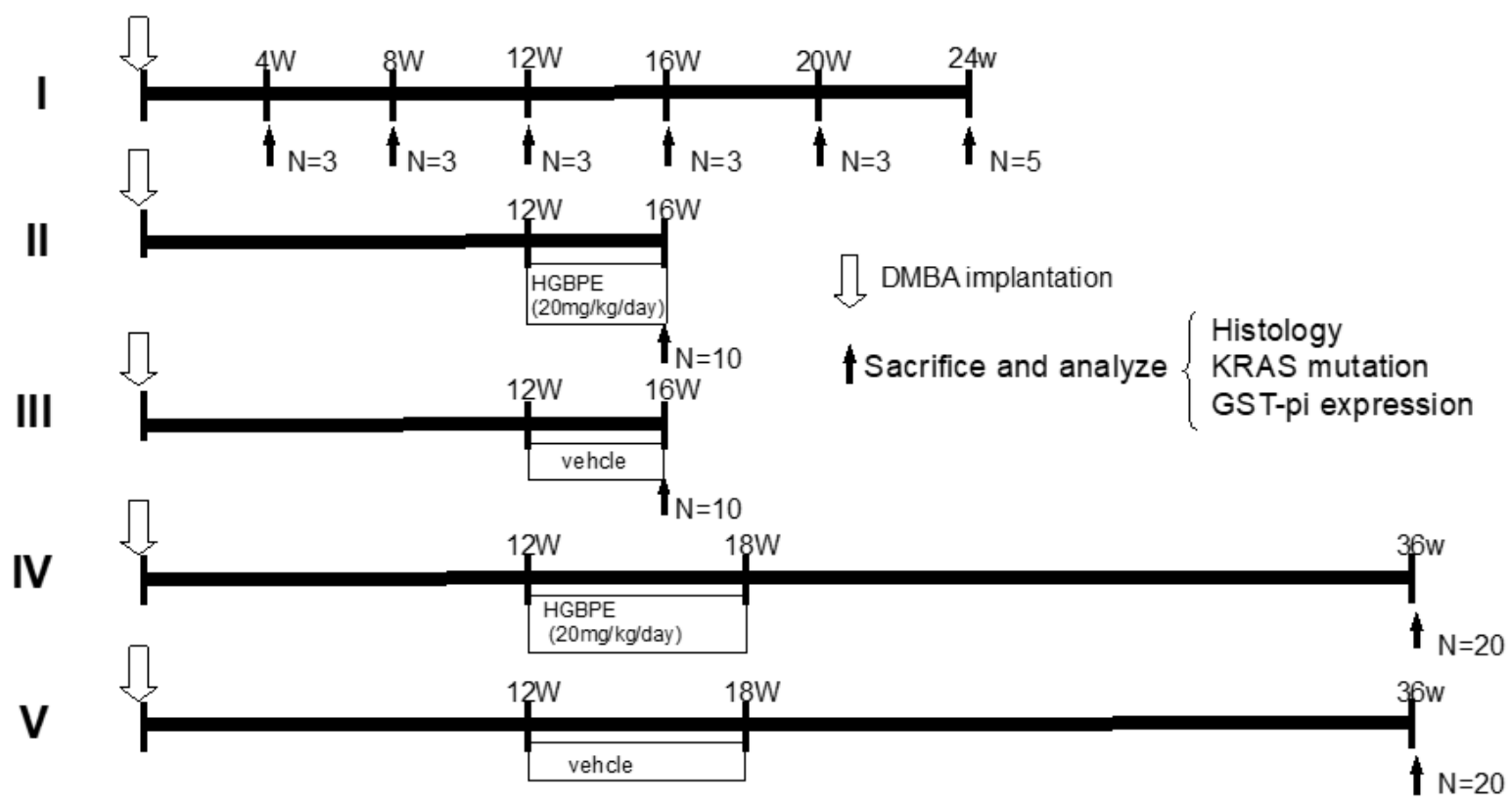

Figure 1

Procedure of DMBA induced pancreas carcinogenesis in rats and its chemoprevention protocol using GSTP1 inhibitor, HGBPE. (A) Implantation of DMBA into the pancreatic head. Rats were anesthetized by intramuscular administration of $10 \mathrm{ml} / \mathrm{kg}$ of the mixture of ketamine hydrochloride $(50 \mathrm{mg} / \mathrm{ml})$ and xylazine hydrochloride $(20 \mathrm{mg} / \mathrm{ml})$ mixed at 7:3 (a), and then the abdomen was incised through a midline incision (b), and the pancreatic head (arrow) was exposed (c). A pocket was created in the pancreatic 
head, 5 mg of DMBA was implanted and then sutured with 6-0 Prolene (d). (B) Structure of 01-Hexadecyly-glutamyl-S-benzylcysteinyl-D-phenylglycine Ethylester (HGBP). Glutathione skeleton indicated by the area enclosed by the line is the substrate of GST, which binds to the glutathione binding site of GST-pi and inhibits the GST-pi activity. (C) Group I, rats were sacrificed and analyzed after 4, 8, 12, 16, 20, and 24 weeks of DMBA implantation; group II, rats received $20 \mathrm{mg} / \mathrm{kg}$ of HGBP intraperitoneally for four consecutive weeks starting at week 12 after DMBA implantation and were sacrificed and analyzed 16 weeks after DMBA implantation; group III, rats received vehicles intraperitoneally for four consecutive weeks starting at week 12 after DMBA implantation and were sacrificed and analyzed 16 weeks after DMBA implantation; Group IV, rats received $20 \mathrm{mg} / \mathrm{kg}$ of HGBP intraperitoneally for six consecutive weeks starting at week 12 after DMBA implantation and were sacrificed and analyzed 36 weeks after DMBA implantation; group $V$ was administered vehicle intraperitoneally for six consecutive weeks and analyzed after 36 weeks of DMBA implantation; group VI was administered vehicle intraperitoneally for six consecutive weeks and analyzed after 36 weeks of DMBA implantation. 


\section{A}

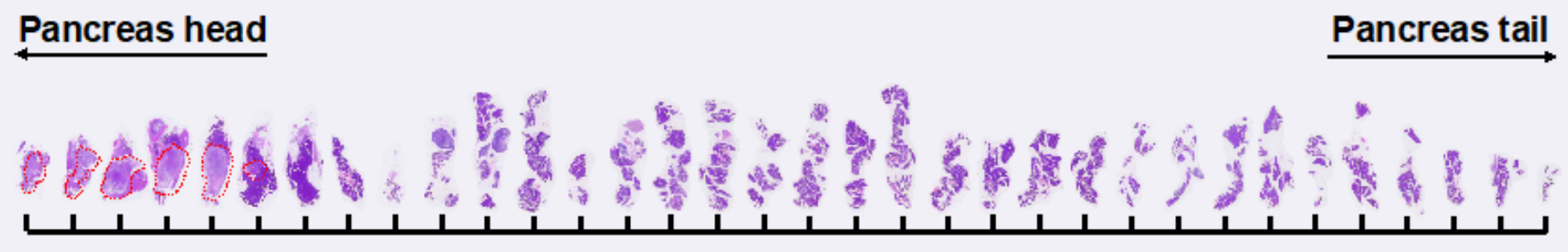

$0 \begin{array}{llllllllllll}1 & 2 & 3 & 4 & 5 & 6 & 7 & 8 & 9 & 1011 & 121314151617181920212223242526272829 & 30313233\end{array}$
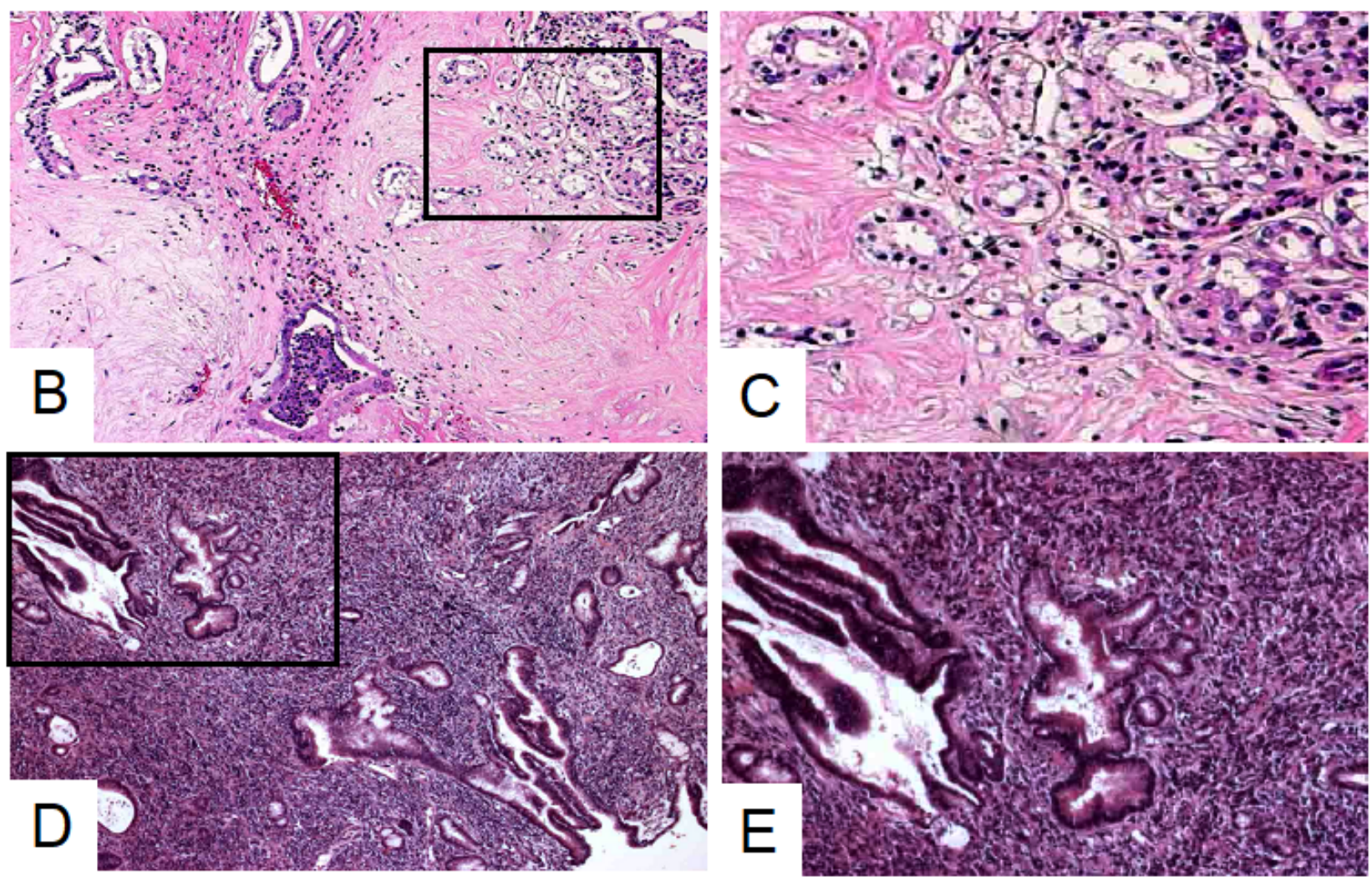

Figure 2

Pathological examination of ductal complex and adenocarcinoma in pancreas tissue of rats treated with DMBA for 14 weeks (group I) (A) Representative HE-stained image of a whole rat pancreas 24 weeks after DMBA implantation. The pancreatic heads were separated at $1 \mathrm{~mm}$ intervals perpendicular to the long axis of the pancreas, each of which was paraffin-embedded and cut out, and HE staining was performed. A white nodule was formed at $6 \mathrm{~mm}$ from the pancreatic head, while no lesion was found in the caudal side of the head. (B-C) HE-stained images of the Ductal complex with weak (x50, B) and strongly magnified $(x 100, C)$. Ductal complex consists of cells with atypical nuclei and cytoplasm, enlarged nuclei and chromatin aggregates, and dense areas of micropancreatic ducts with no tendency to infiltrate. (D-E) 
HE-stained images of adenocarcinoma. Weakly magnified (x50, D) and strongly magnified (x100, E). adenocarcinoma showed evident nuclear structural atypia and stratification.

A

Normal

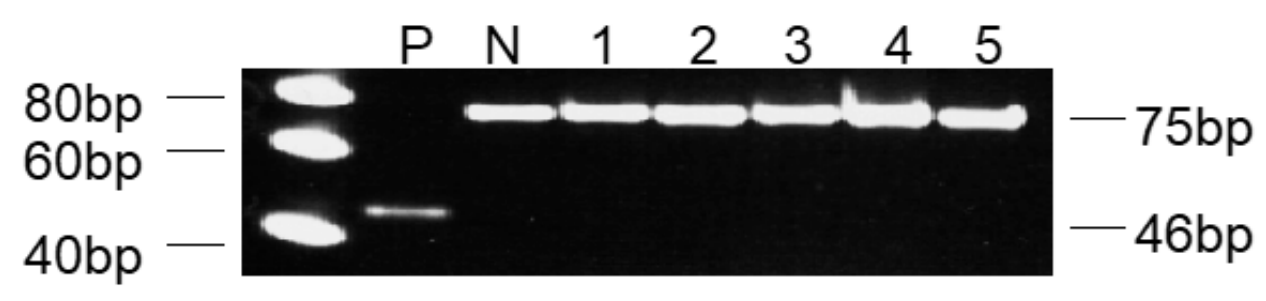

Ductal complex
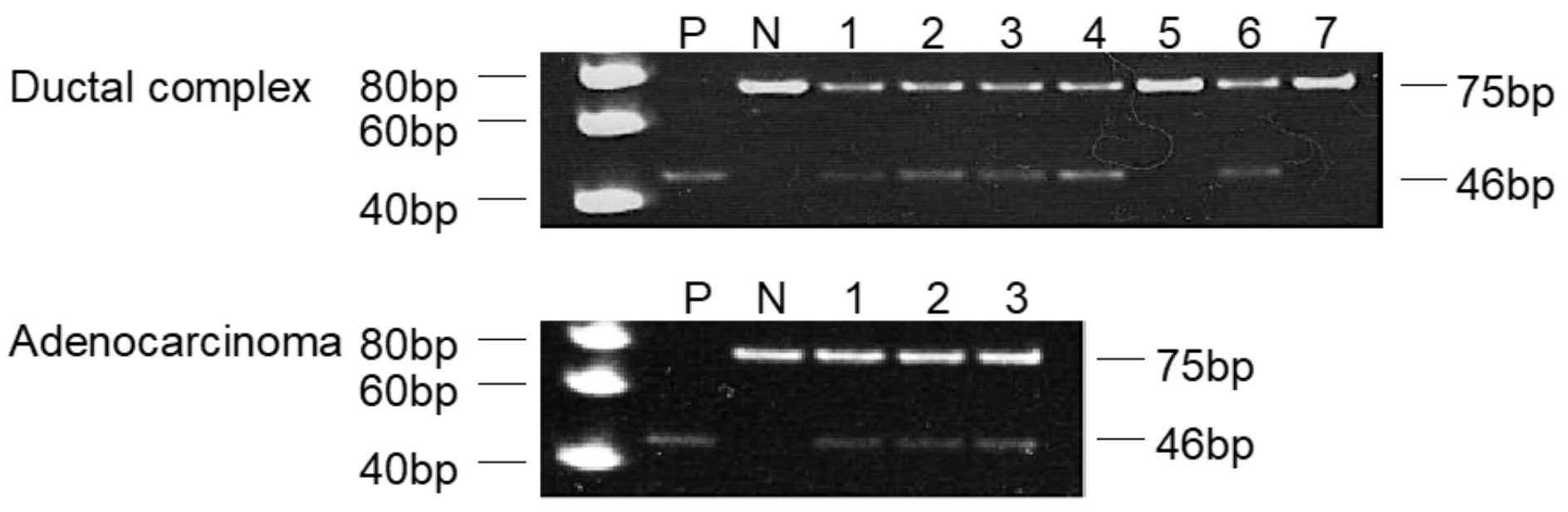

Incidence of K-ras mutation

\begin{tabular}{lll}
\hline Normal & $0 / 5$ & $(0 \%)$ \\
Ductal complex & $5 / 7$ & $(71.4 \%)$ \\
Adenocarcinoma & $3 / 3$ & $(100 \%)$ \\
\hline
\end{tabular}

Figure 3

Analysis of K-ras gene mutations in ductal complex and adenocarcinoma (group 1) using polymerase chain reaction-restriction fragment length polymorphism (PCR-RFLP). (A) Results of 2-step PCR-RFLP analysis of K-ras gene at codon 12. DNA extracts from tumor samples were submitted to double PCR amplification after Mval and Hphl enzymatic digestion. Wild-type K-ras gene at codon 12 is not digested by the restriction enzyme $\mathrm{Hph}$ I, resulting in a $75 \mathrm{bp}$ fragment; however, KRAS gene with point mutations in codon 12 can be digested, resulting in a 46-bp fragment. P, codons 12 mutated DNA were used as a 
positive control. N, wild-type K-ras DNA used as negative controls. (B) Incidence of K-ras gene mutation from tumor samples. Positivity of K-ras gene mutation was $0 \%$ in the normal duct, $71.4 \%$ in ductal complex, and $100 \%$ in adenocarcinoma.

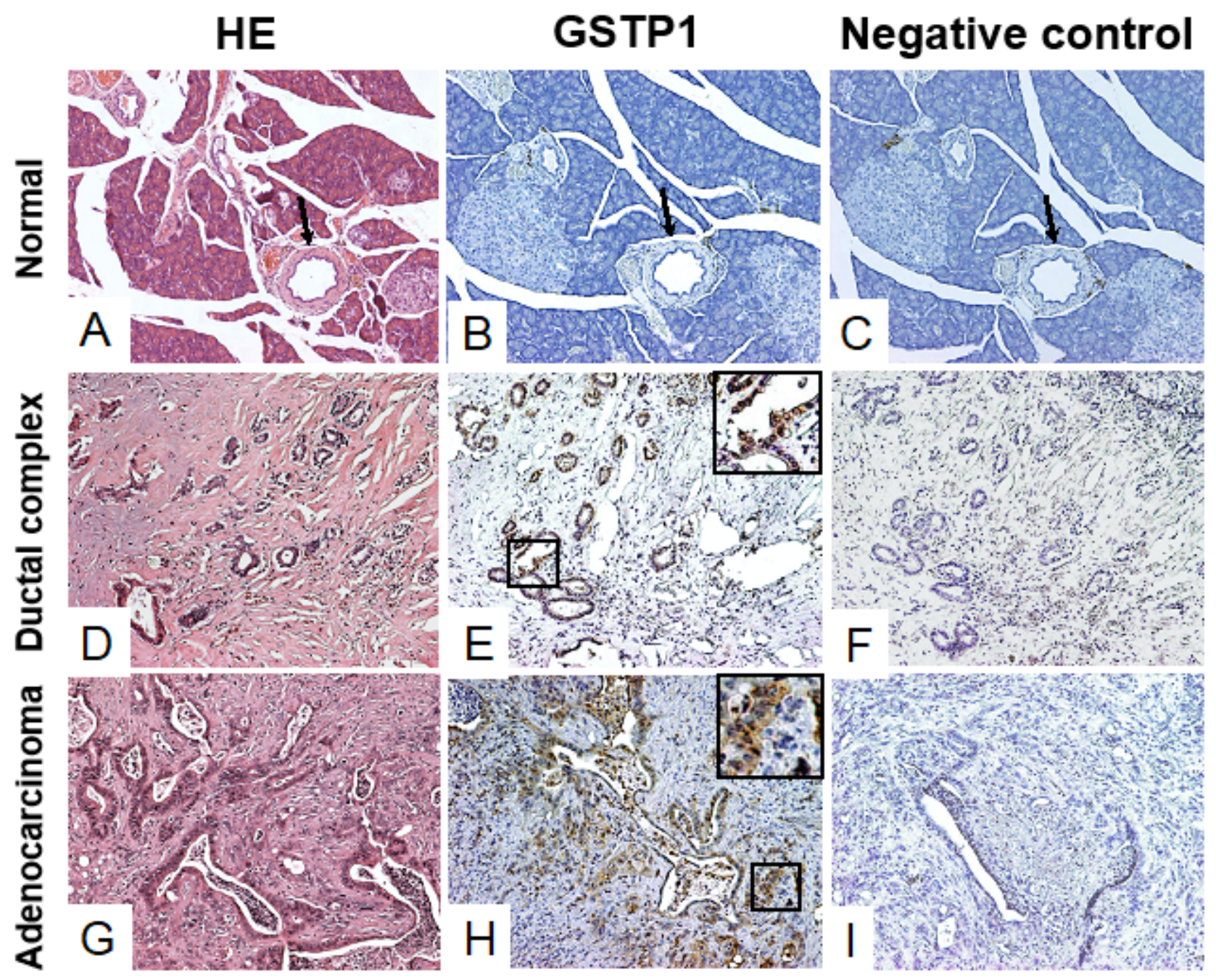

Figure 4

GST-pi immunohistochemistry of ductal complex and adenocarcinoma of rat pancreas. Representative immunohistochemistry of specimens from untreated normal rat pancreas (A-C), ductal complex from rat pancreas after 16 weeks of DMBA implantation (D-F), and pancreas adenocarcinoma after 24 weeks of DMBA implantation (G-I); HE staining (A, D, G), GST-pi staining (B, E, H), and negative control (without primary antibody) $(C, F, I)$, respectively. No staining for GST-pi was found in the normal pancreatic ducts (arrows). Clear GST-pi staining in the cytoplasm were observed in enlarged images of the corresponding boxed areas indicated $(E, H)$. 